\title{
APPROXIMATE DISTRIBUTIONS OF NOISE POWER MEASUREMENTS*
}

\author{
BY \\ WALTER FREIBERGER AND ULF GRENANDER \\ Brown University
}

Summary. The frequency functions of certain spectral estimates are studied analytically and numerically. An approximation is obtained for the case of a Poisson weight function and compared to the true distribution. The eigenvalues of products of Toeplitz matrices play a crucial role in the sampling theory of quadratic forms; an approximation to their distribution is discussed and its accuracy studied numerically. This leads to approximate probability densities which are thought to be valid for moderate or even small sample sizes.

1. Introduction. Let $x_{t}$ be stationary Gaussian noise with the power spectral density $f(\lambda)$. The parameter $t$ takes on integral values only, $t=\cdots-1,0,1, \cdots$. Given a finite sample $x_{1}, x_{2}, \cdots, x_{n}$ we want to estimate $f(\lambda)$, using a quadratic form $Q$ of the type

$$
Q=\frac{1}{n} \sum_{\nu, \mu=1}^{n} w_{\nu-\mu} x_{\nu} x_{\mu}
$$

where

$$
w_{k}=\frac{1}{2 \pi} \int_{-\pi}^{\pi} e^{i k \lambda} w(\lambda) d \lambda .
$$

Here the weight function $w(\lambda)$ defines the spectral window and satisfies

$$
\begin{gathered}
w(\lambda) \geq 0 \\
\text { 2) } \frac{1}{2 \pi} \int_{-\pi}^{\pi} w(\lambda) d \lambda=1 .
\end{gathered}
$$

If the sample size $n$ is large, it is known that $Q$ has an asymptotically Gaussian distribution with parameters that can be expressed simply in terms of $f(\lambda)$ and $w(\lambda)$; see Grenander and Rosenblatt (1957), p. 134 [1]. On this basis a choice between various a priori possible estimates can be rationally made, in accordance with the well-known relation between the band-width and variance of a spectral estimate.

A considerable number of particular weight functions has been suggested in the statistical literature. The usual procedure is to choose an even, periodic weight function $v(\lambda)$, subject to the above conditions (3), and to form

$$
w(\lambda)=\frac{1}{2}\left[v\left(\lambda-\lambda_{0}\right)+v\left(\lambda+\lambda_{0}\right)\right]
$$

where $\lambda_{0}$ is the frequency at which we want to determine $f\left(\lambda_{0}\right)$. If the Fourier coefficients of $v(\lambda)$ are denoted by $v_{k}$ then clearly $w_{k}=v_{k} \cos k \lambda_{0}$.

${ }^{*}$ Received October 22, 1958. The work described here has been partly supported by the U.S. Army Signal Corps, under Contract DA-SC-78130. 
We will be concerned with two particular cases. The first is the rectangular weight function

$$
v(\lambda)= \begin{cases}\frac{\pi}{h}, & |\lambda|<h \\ 0, & |\lambda|>h .\end{cases}
$$

It is of course desirable to keep the bandwidth parameter $h$ small, but it must not be chosen so small that the variance becomes excessively large.

The other is the Poisson weight function

$$
v(\lambda)=\left.\frac{1-\rho^{2}}{\mid 1-\rho e^{i \lambda}}\right|^{2}=\frac{1-\rho^{2}}{1-2 \rho \cos \lambda+\rho^{2}}, \quad 0 \leq \rho<1,
$$

where values of $\rho$ close to one correspond to narrow spectral windows.

The Fourier coefficients $v_{k}$ are in the first case $\sin k h / k h$ and in the second $\rho^{|k|}$. Both these windows are convenient to deal with and there are reasons to believe they have good sampling properties.

The distribution theory for spectral estimates is fairly complicated. It is true that one can write down immediately the characteristic function $\varphi(z)$ of $Q$ as

$$
\varphi(z)=\prod_{\nu=1}^{n}\left(1-2 \frac{i z \lambda_{v}}{n}\right)^{-1 / 2},
$$

where the $\lambda_{v}$ are the eigenvalues (always real) of the matrix product $R W$. Here $R$ is the covariance matrix

$$
\begin{aligned}
R & =\left\{r_{\nu-\mu} ; \quad \nu, \mu=1,2, \cdots, n\right\} \\
& =\left\{\frac{1}{2 \pi} \int_{-\pi}^{\pi} e^{i(\nu-\mu) \lambda} f(\lambda) d \lambda ; \quad \nu, \mu=1,2, \cdots, n\right\}
\end{aligned}
$$

and

$$
W=\left\{w_{\nu-\mu} ; \quad \nu, \mu=1,2, \cdots n\right\} .
$$

The expression (7) is of little immediate practical use since (a) the eigenvalues are in general very difficult to obtain numerically and (b) it is very hard to get the frequency function $g(x)$ of $Q$ by numerical Fourier inversion. Therefore, one is almost forced to resort to approximations, of which the most widely discussed is the Gaussian one mentioned above.

Recently attention has been focused on the need for sharper approximations, valid for moderate sample sizes. One such approximation can be obtained by applying known results from the theory of Toeplitz forms. Indeed, one knows that the $\lambda_{v}$ behave distributionwise approximately as the values of the function $w(\lambda) f(\lambda)$, viz.

$$
\lim _{n \rightarrow \infty} \frac{1}{n}\left\{\text { number of } \lambda_{\nu} \leq \mu\right\}=\frac{1}{2 \pi} \text { meas }\{\lambda \mid f(\lambda) w(\lambda) \leq \mu\} ;
$$

see Grenander and Szegö (1958), Chap. 8 [2]. This implies that we have approximately

$$
\log \varphi(z)=-\frac{1}{2} \sum_{\nu=1}^{n} \log \left(1-\frac{2 i z \lambda_{\nu}}{n}\right) \cong-\frac{n}{4 \pi} \int_{-\pi}^{\pi} \log \left[1-\frac{2 i z f(\lambda) w(\lambda)}{n}\right] d \lambda .
$$


Before entering into a discussion of how (11) can be used to determine the approximate frequency function of $Q$, one important observation will be made. It is clear that a spectral estimate will be useful only if it resolves the spectrum well enough so that $w(\lambda)$ is peaked around the frequency of interest $\lambda_{0}$. If the spectral window is narrow, measured in terms of a typical frequency of the stochastic process, then the values of the distributions of the two functions $f(\lambda) w(x)$ and $f\left(\lambda_{0}\right) w(\lambda)$ are almost identical, so that little is changed in (11) if we substitute the second function for the first one.

Hence the stochastic variable $Q / f\left(\lambda_{0}\right)$ will have approximately the characteristic function

$$
E \exp \left[i z Q / f\left(\lambda_{0}\right)\right] \cong \varphi_{a}(z)=\exp \left\{-\frac{n}{4 \pi} \int_{-\pi}^{\pi} \log \left[1-\frac{2 i z w(\lambda)}{n}\right] d \lambda\right\} .
$$

This distribution apparently does not depend upon the (unknown) spectral density $f(\lambda)$, so that we can use it to construct approximate confidence limits $f_{1}^{*}\left(\lambda_{0}\right)$ and $f_{2}^{*}\left(\lambda_{0}\right)$. Indeed, $f_{i}^{*}\left(\lambda_{0}\right)=Q / x_{i}$ with

$$
\int_{x_{1}}^{x_{2}} g_{a}(x) d x=p
$$

where $p$ is the confidence coefficient and $g_{a}(x)$ is the frequency function corresponding to $\varphi_{a}(z)$.

A parallel investigation, Grenander, Pollak and Slepian (1959) [3], discussed the approximation (11) for $f(\lambda) \equiv 1$. The approximation mentioned in the last paragraph does not seem to have been studied numerically in the literature. This will be done for a few cases in the following section.

2. Numerical studies. Let us introduce the eigenvalues $\lambda_{k}^{\prime}(k=1,2, \cdots, n)$ of the matrix $f\left(\lambda_{0}\right) W$; as mentioned above, these eigenvalues can be expected to have about the same distribution as $\lambda_{k}$. In studying this approximation numerically we are constrained by the limitations of our computing facilities. To avoid excessive computing effort, we have chosen $n=20$. This number may not correspond to situations met frequently in practice, but it is hoped that the information gained will be of some general interest.

The spectral density was chosen as

$$
f(\lambda)=102+22 \cos 2 \lambda+20 \cos 4 \lambda,
$$

a graph of which is shown in Fig. 1(1). The value of $f(\lambda)$ at $\lambda_{0}=0$ is $f(0)=144$; the Poisson weight function (6) was used with $\rho=.7, .9$ and .95 . The eigenvalues $\lambda_{k}$ and $\lambda_{k}^{\prime}$ are given in Table 1 .

The statistical distribution of the noise-power estimates will depend mainly upon the largest eigenvalues and the table shows that the error in these is of the order of a few per cent. We have also computed the exact frequency function $g(x)$ and compared it with the frequency function corresponding to the eigenvalues $\lambda_{k}^{\prime}$; see Figs. 2-4.

The frequency functions have been computed from the expression

$$
g(x)=\frac{1}{\pi} \sum_{\nu=1}^{10}(-1)^{\nu+1} \int_{\left(2 \lambda_{2 y-1}\right)-1}^{\left(2 \lambda_{z \nu}\right)^{-1}} e^{-y x} \prod_{j=1}^{20}\left(1-2 y \lambda_{i}\right)^{-1 / 2} d y
$$

and the corresponding formula with the $\lambda_{v}$ replaced by the $\lambda_{v}^{\prime}$. This expression was given by Slepian (1958) [4]. To eliminate singularities at the ends of the intervals of 


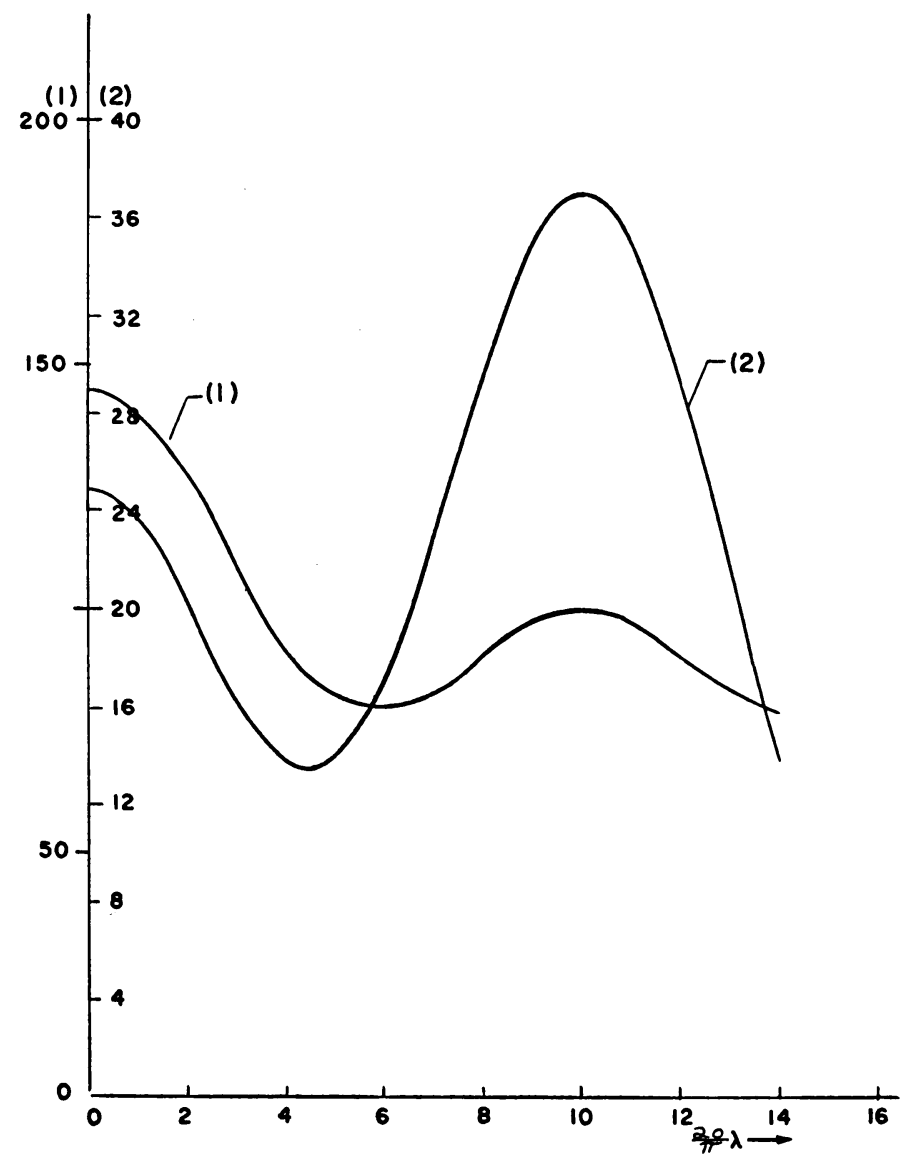

FIG. 1

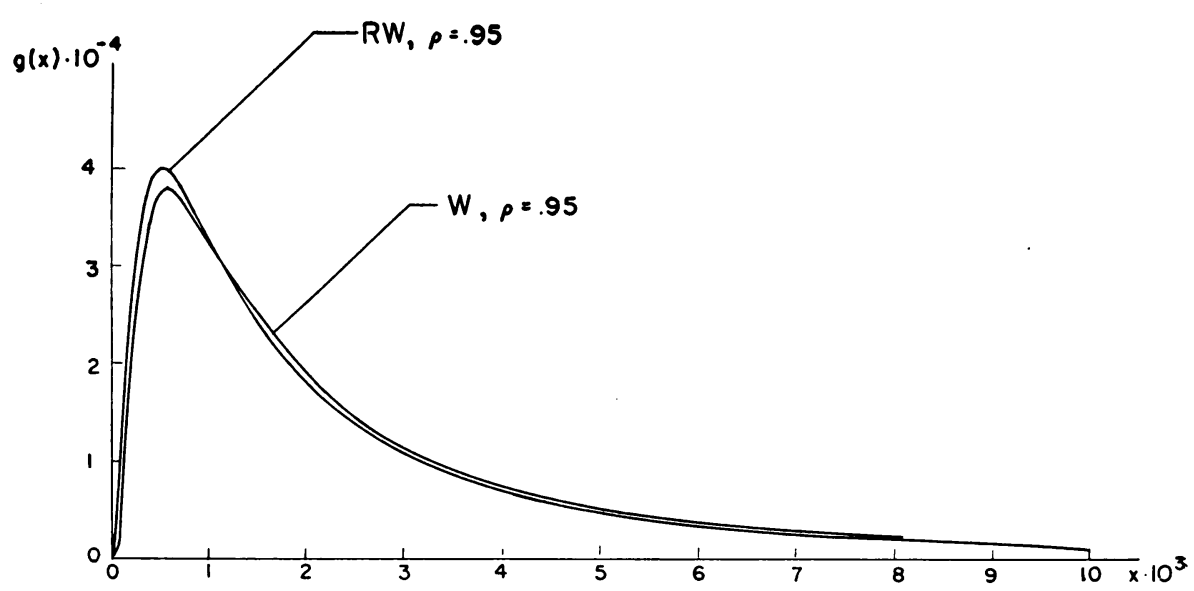

FIG. 2 
TABLE 1

\begin{tabular}{|c|c|c|c|c|c|c|}
\hline \multirow[t]{2}{*}{$k$} & \multicolumn{2}{|c|}{$\rho=.95$} & \multicolumn{2}{|c|}{$\rho=.9$} & \multicolumn{2}{|c|}{$\rho=.7$} \\
\hline & $\begin{array}{c}\lambda_{k} \\
2078.9\end{array}$ & $\begin{array}{c}\lambda_{k}^{\prime} \\
2114.6\end{array}$ & $\begin{array}{c}\lambda_{k} \\
1596.0\end{array}$ & $\begin{array}{c}\lambda_{k}^{\prime} \\
1618.3\end{array}$ & $\begin{array}{c}\lambda_{k} \\
7233\end{array}$ & $\begin{array}{c}\lambda_{k}^{\prime} \\
729\end{array}$ \\
\hline 2 & 386.9 & 405.4 & 552.1 & 576.0 & 529.4 & 547.3 \\
\hline 3 & 124.6 & 134.1 & 220.2 & 237.5 & 353.4 & 379.0 \\
\hline 4 & 57.1 & 64.2 & 108.8 & 122.4 & 232.0 & 260.9 \\
\hline 5 & 31.6 & 37.6 & 62.1 & 73.9 & 155.2 & 185.0 \\
\hline 6 & 19.6 & 24.8 & 39.0 & 49.5 & 107.0 & 136.4 \\
\hline 7 & 13.0 & 17.7 & 26.2 & 35.7 & 76.3 & 104.3 \\
\hline 8 & 9.2 & 13.4 & 18.6 & 27.2 & 56.2 & 82.7 \\
\hline 9 & 6.8 & 10.7 & 13.8 & 21.6 & 42.8 & 67.5 \\
\hline 10 & 5.2 & 8.8 & 10.7 & 17.7 & 33.9 & 56.6 \\
\hline 11 & 4.3 & 7.3 & 8.7 & 15.0 & 27.9 & 48.5 \\
\hline 12 & 3.6 & 6.3 & 7.3 & 13.0 & 23.9 & 42.5 \\
\hline 13 & 3.2 & 5.6 & 6.5 & 11.5 & 21.2 & 37.9 \\
\hline 14 & 2.9 & 5.0 & 5.9 & 10.4 & 19.5 & 34.3 \\
\hline 15 & 2.7 & 4.6 & 5.6 & 9.5 & 18.5 & 31.5 \\
\hline 16 & 2.6 & 4.3 & 5.4 & 8.9 & 18.0 & 29.5 \\
\hline 17 & 2.6 & 4.0 & 5.3 & 8.3 & 17.7 & 27.9 \\
\hline 18 & 2.6 & 3.9 & 5.3 & 8.1 & 17.6 & 26.8 \\
\hline 19 & 2.6 & 3.7 & 5.3 & 7.8 & 17.6 & 26.1 \\
\hline 20 & 2.6 & 3.7 & 5.3 & 7.6 & 17.6 & 25.3 \\
\hline
\end{tabular}

integration, a change of variable

$$
y=c_{\nu} \cos \pi x+d_{\nu},
$$

with

$$
c_{v}=\frac{1}{2}\left(y_{2 v+1}-y_{2 v}\right), \quad d_{v}=\frac{1}{2}\left(y_{2 v+1}+y_{2 v}\right), \quad y_{v}=\frac{1}{2 \lambda_{v}}
$$

is effected and the resulting integrals are evaluated by a Newton-Coates five-point formula.

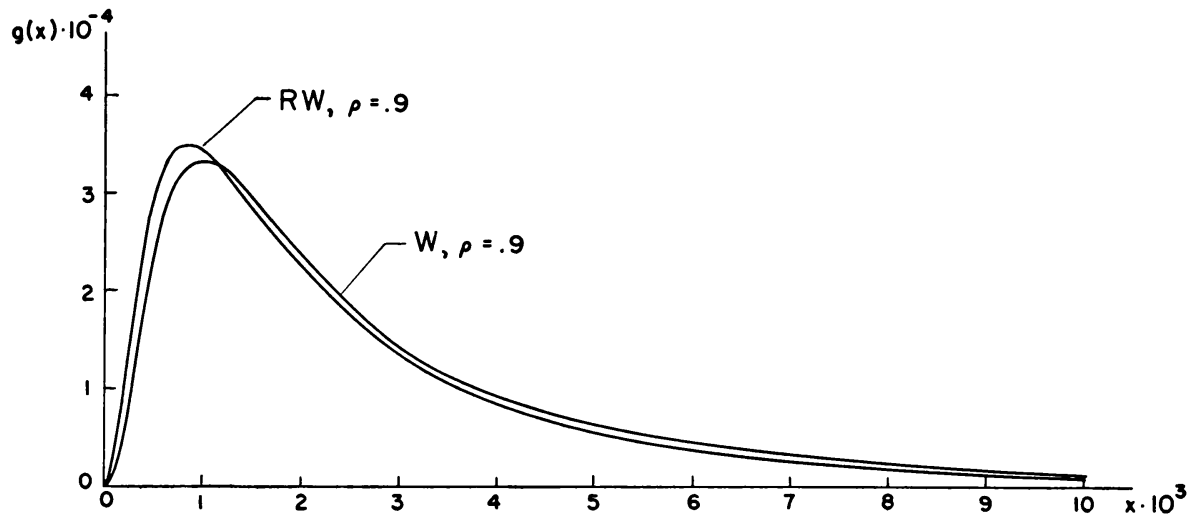

Fig. 3 


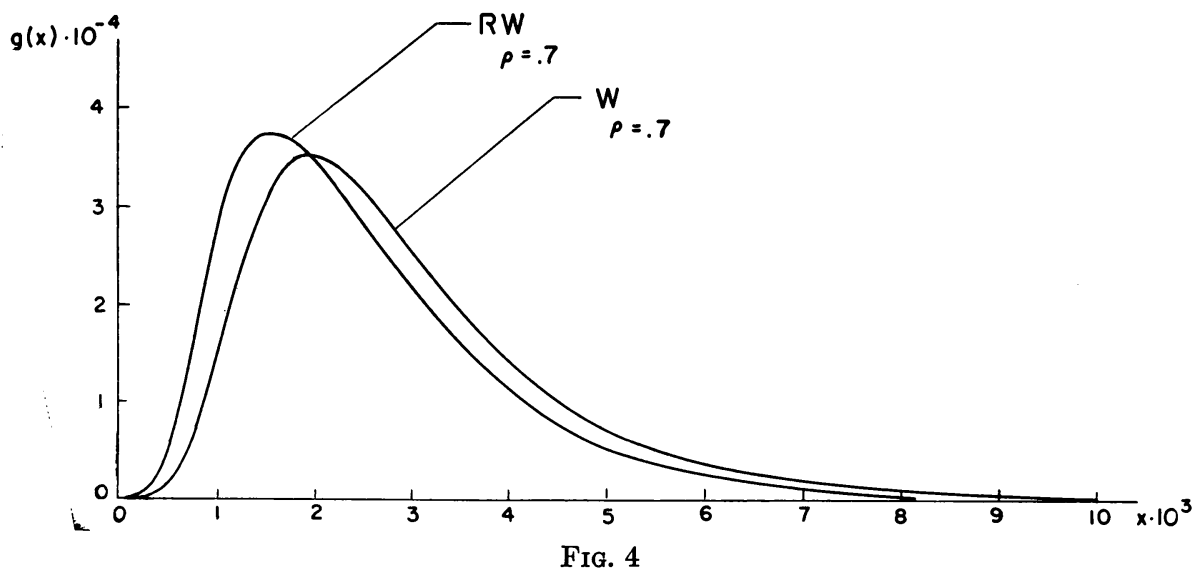

Because of the proximity of the eigenvalues, which is characteristic for matrices of this type, the classical iteration procedure broke down. Instead, the Jacobi diagonalisation method was modified to apply to (non-symmetric) matrices which are products of two symmetric matrices; see Kalker (1958) [5]. The computations were carried out on an IBM 650 computer and took of the order of a few hours for the determination of the eigenvalues and of each frequency function.

It is obvious from the graphs given below that the approximation works well for the narrow windows $\rho=.95, .90, .70$. If the weight function is flat one will not expect the approximation to be adequate and in the cases $\rho=.5$ and .3 we give only the $\lambda_{k}^{\prime}$ in Table 2 and the corresponding frequency functions in Figs. 5 and 6 . This table may be of interest to a reader who wishes to investigate wide spectral windows.

TABLE 2

\begin{tabular}{r||r||c}
\hline \hline$k$ & $\rho=.5$ & $\rho=.3$ \\
\hline & $\lambda_{k^{\prime}}$ & $\lambda_{k}{ }^{\prime}$ \\
1 & 416.4 & 264.1 \\
2 & 375.5 & 254.6 \\
3 & 322.6 & 240.3 \\
4 & 269.1 & 223.1 \\
5 & 221.9 & 204.6 \\
6 & 183.3 & 186.2 \\
7 & 152.6 & 168.9 \\
8 & 128.7 & 153.4 \\
9 & 110.2 & 139.4 \\
10 & 95.6 & 127.4 \\
11 & 84.2 & 117.2 \\
12 & 75.3 & 108.4 \\
13 & 68.3 & 101.1 \\
14 & 62.8 & 95.0 \\
15 & 58.3 & 90.0 \\
16 & 54.9 & 86.0 \\
17 & 52.3 & 82.8 \\
18 & 50.4 & 80.5 \\
19 & 49.0 & 78.8 \\
20 & 48.2 & 77.9 \\
& & \\
\hline
\end{tabular}




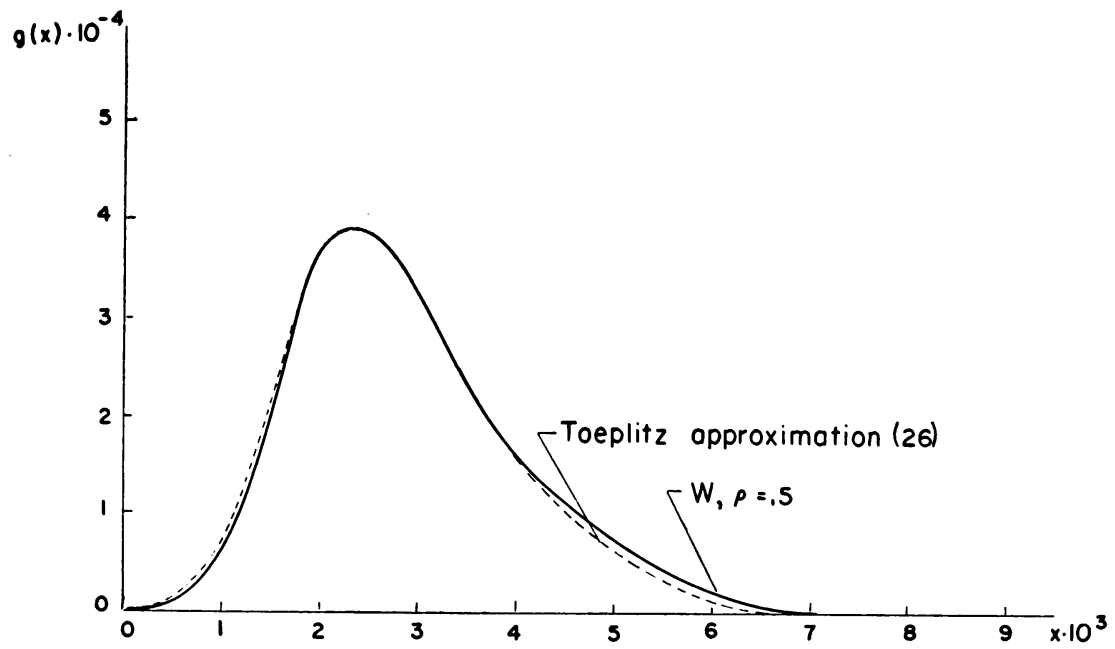

FIG. 5

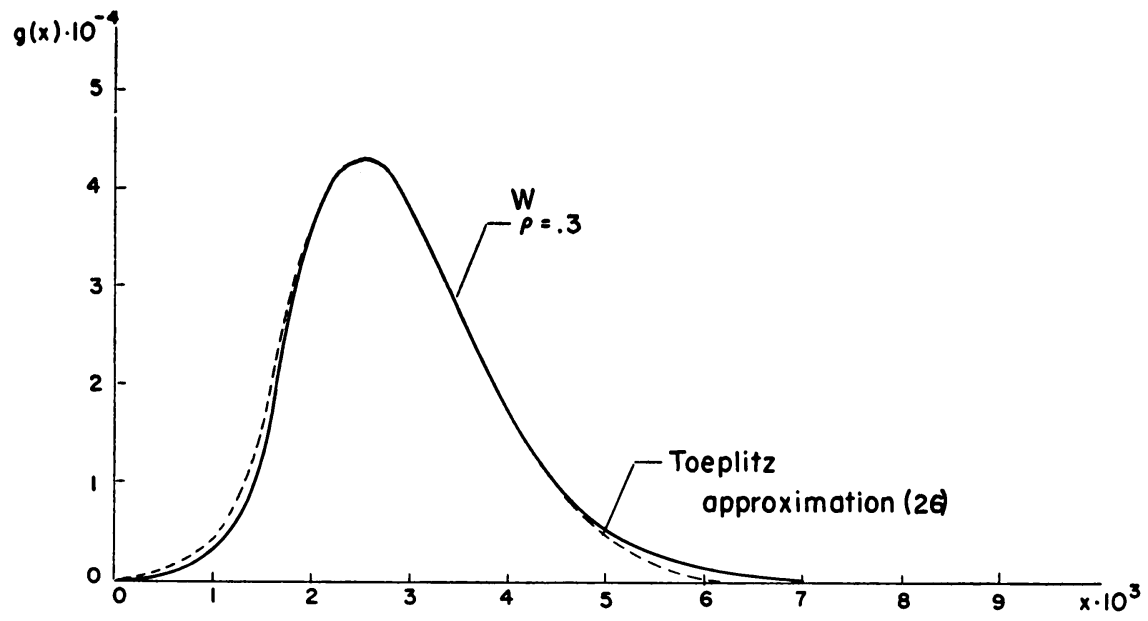

Fig. 6

For the rectangular window (5) the analogous quantities have been computed for the spectral density

$$
f(\lambda)=19+6 \cos \lambda-10 \cos 2 \lambda+2 \cos 3 \lambda+8 \cos 4 \lambda
$$

the graph of which is shown in Fig. 1(2). The value of $f(\lambda)$ at the center $\lambda_{0}=3 / 10 \pi$ of the windows is $f\left(\lambda_{0}\right)=17.2$. The weight function (5) was used with

$$
h=\frac{\pi}{40}, \quad \frac{\pi}{20}, \quad 3 \frac{\pi}{40}, \quad \frac{\pi}{8}, \quad \frac{\pi}{4} .
$$

For the first three of these values we have Table 3 . 
TABLE 3

\begin{tabular}{|c|c|c|c|c|c|c|}
\hline$k$ & \multicolumn{2}{|c|}{$h=\pi / 40$} & \multicolumn{2}{|c|}{$h=\pi / 20$} & \multicolumn{2}{|c|}{$h=3 \pi / 40$} \\
\hline & $\begin{array}{r}\lambda_{k} \\
?\end{array}$ & $\begin{array}{c}\lambda_{k}{ }^{\prime} \\
161^{2}\end{array}$ & $\lambda_{k}$ & $\begin{array}{c}\lambda_{k}^{\prime} \\
\end{array}$ & $\lambda_{k}$ & $\lambda_{k^{\prime}}$ \\
\hline 1 & 176.2 & 161.2 & 147.5 & 135.3 & 118.9 & 107.2 \\
\hline 2 & 166.6 & 160.9 & 140.9 & 134.7 & 113.7 & 106.7 \\
\hline 3 & 13.8 & 10.9 & 44.0 & 35.2 & 68.2 & 56.5 \\
\hline 4 & 11.6 & 10.7 & 37.2 & 34.9 & 58.5 & 56.2 \\
\hline 5 & .175 & .1 & 2.7 & 1.9 & 12.1 & 8.7 \\
\hline 6 & .126 & .1 & 2.0 & 1.7 & 9.0 & 8.0 \\
\hline 7 & 0 & 0 & .1 & 0 & .6 & 0 \\
\hline 8 & 0 & 0 & 0 & 0 & .3 & 0 \\
\hline 9 & $\downarrow$ & $\downarrow$ & 0 & 0 & 0 & 0 \\
\hline 10 & & & $\downarrow$ & $\downarrow$ & 0 & 0 \\
\hline 11 & & & & & & \\
\hline 12 & & & & & $\downarrow$ & $\downarrow$ \\
\hline & & & & & & \\
\hline
\end{tabular}

While the approximation still seems adequate it is interesting to note that the relative errors of the largest eigenvalues are now somewhat larger than for the Poisson weight function. The explanation for this may be found in the local behavior of the spectral densities at the frequencies of interest.

The resulting frequency functions are represented in Figs. 7-9. We also give the values of $\lambda_{k}^{\prime}$ for $h=\pi / 8$ and $\pi / 4$, in Table 4 .

TABLE 4

\begin{tabular}{r||c||c}
\hline \multicolumn{1}{r||}{$k$} & $h=\pi / 8$ & $h=\pi / 4$ \\
\hline & $\lambda_{k}{ }^{\prime}$ & $\lambda_{k}{ }^{\prime}$ \\
1 & 46.0 & 30.9 \\
2 & 45.7 & 26.6 \\
3 & 29.7 & 23.7 \\
4 & 27.8 & 21.1 \\
5 & 17.0 & 19.6 \\
6 & 16.0 & 18.9 \\
7 & 14.9 & 18.4 \\
8 & 14.9 & 18.4 \\
9 & 14.9 & 18.2 \\
10 & 14.9 & 18.2 \\
11 & 14.9 & 16.6 \\
12 & 14.9 & 16.6 \\
13 & 14.8 & 16.5 \\
14 & 14.6 & 16.4 \\
15 & 14.4 & 16.2 \\
16 & 14.3 & 15.8 \\
17 & 11.6 & 14.3 \\
18 & 10.6 & 10.4 \\
19 & 4.8 & 3.9 \\
20 & 3.3 & 3.5 \\
& & \\
\hline
\end{tabular}




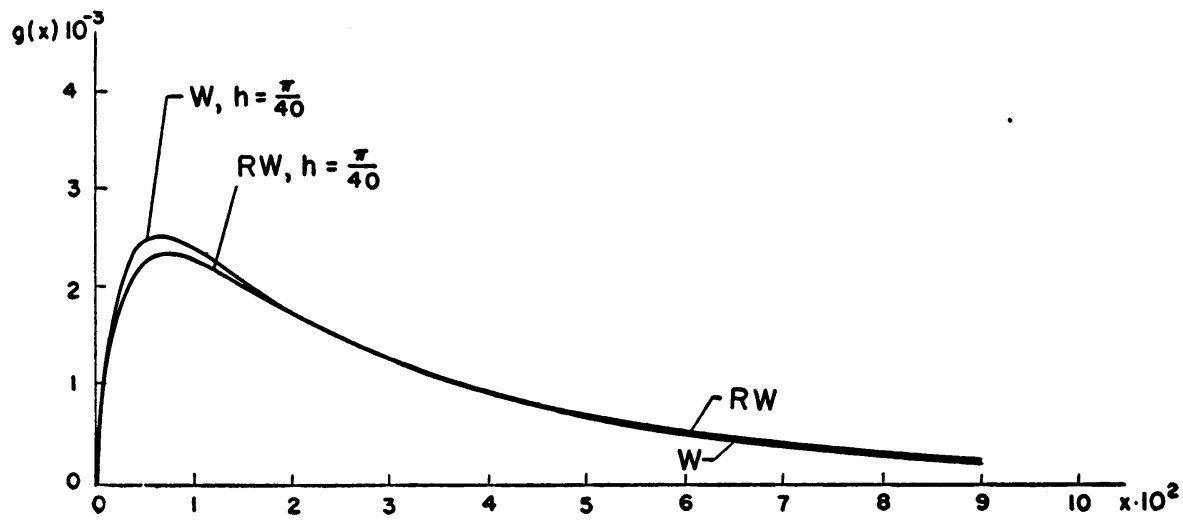

Fig. 7

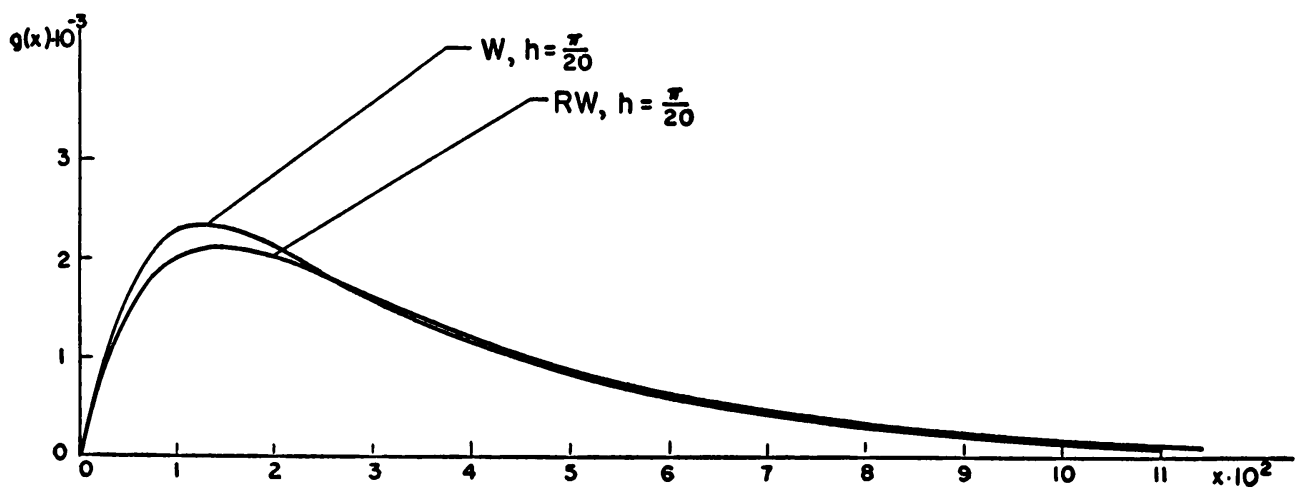

Fig. 8

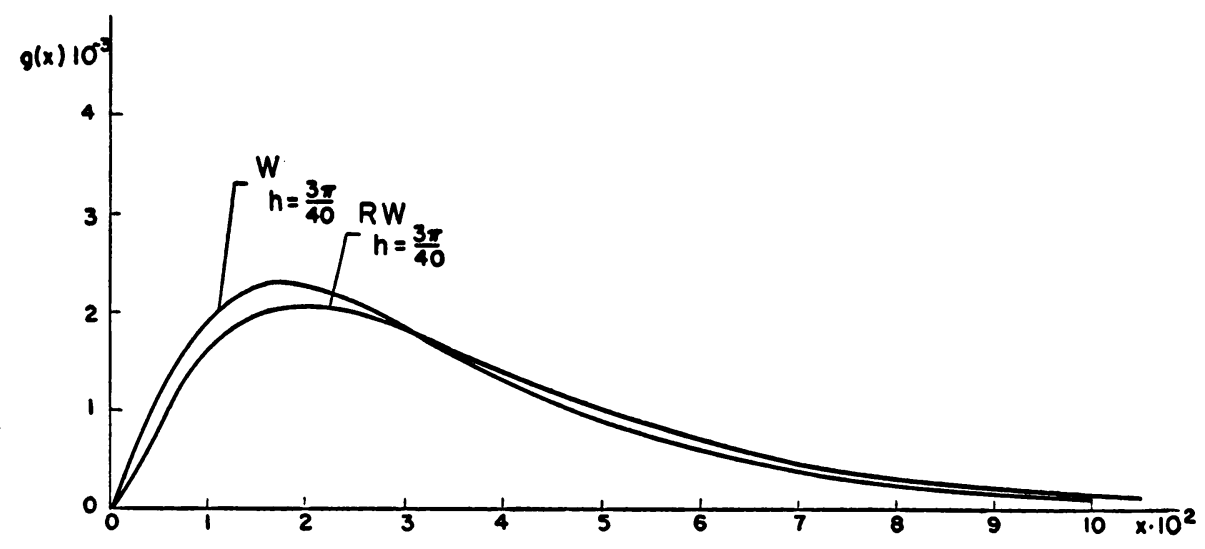

Fig. 9

Similar observations to those made in the case of the Poisson weight function seem to apply here.

3. Some closed-form approximations. We have seen in the last section that if the spectral window is sufficiently narrow, little accuracy is lost if we regard $f(\lambda)$ constant 
throughout the interval $(-\pi, \pi)$. If this is done, and if $f(\lambda)$ is normed to 1 , we can use Eq. (12) to give us the approximate expression for the characteristic function

$$
\varphi_{\alpha}(z)=\exp \left[-\frac{n}{2} \psi(z)\right],
$$

where

$$
\psi(z)=\frac{1}{2 \pi} \int_{0}^{2 \pi} \log \left[1-\frac{2 i z w(\lambda)}{n}\right] d \lambda .
$$

For the case of a rectangular window this equation gives us the characteristic function of the well-known type III distribution with

$$
\psi(z)=\frac{h}{\pi} \log \left[1-\frac{2 \pi i z}{n h}\right]
$$

so that

$$
\varphi_{\alpha}(z)=\left[1-\frac{2 \pi i z}{n h}\right]^{-n h / 2 \pi} .
$$

This corresponds to the frequency function

$$
g_{\alpha}(x)=\left(\frac{n h}{\pi}\right)^{h n / 2 \pi} \frac{1}{2^{n n / 2 \pi} \Gamma(h n / 2 \pi)} x^{h n / 2 \pi-1} \exp [-(x n h / 2 \pi)],
$$

an approximation which may in essence be contained in the work of S. O. Rice.

The Toeplitz approximation for a Poisson spectral window can also be expressed in closed form. Indeed, the function

$$
\exp \psi(z)=\exp \frac{1}{2 \pi} \int_{0}^{2 \pi} \log \left[1-\frac{2 i z}{n} \frac{1-\rho^{2}}{\left|1-\rho e^{i \lambda}\right|^{2}}\right] d \lambda
$$

is analytic in $z$ in the plane cut from

$$
z=-\frac{n}{2} \frac{1-\rho}{1+\rho} i \text { to } z=-\frac{n}{2} \frac{1+\rho}{1-\rho} i, \quad \text { see Fig. } 10 .
$$

Noticing that

$$
|\operatorname{Re} \psi(z)| \leq \frac{1}{2} \log \left[1+\frac{4 z^{2}}{n^{2}}\left(\frac{1+\rho}{1-\rho}\right)^{2}\right],
$$

so that $\left|\varphi_{\alpha}(z)\right|$ is dominated by a power of $z$, we can change that contour of integration in the Fourier inversion formula

$$
g_{\alpha}(x)=\frac{1}{2 \pi} \int_{-\infty}^{\infty} e^{-i x z} \varphi(z) d z
$$

to give us

$$
g_{\alpha}(x)=\frac{1}{2 \pi} \oint e^{-i x z} \varphi(z) d z
$$

However

$$
\psi(z)=\frac{1}{2 \pi} \int_{0}^{2 \pi}\left\{\log \left[\left|1-\rho e^{i \lambda}\right|^{2}-\frac{2 i z}{n}\left(1-\rho^{2}\right)\right]-\log \left|1-\rho e^{i \lambda}\right|^{2}\right\} d \lambda .
$$




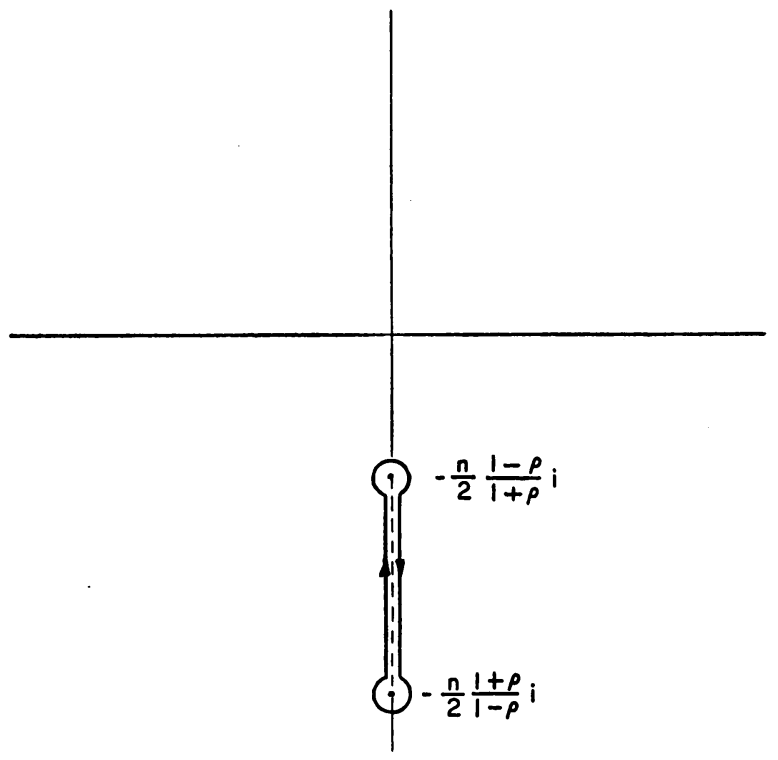

Fig. 10. z-plane.

The last term does not contribute anything to the integral and putting $z=-i v$ we get

$$
\psi(z)=\frac{1}{2 \pi} \int_{0}^{2 \pi} \log \left[B\left|1-R e^{i \lambda}\right|^{2}\right] d \lambda,
$$

where

$$
B\left|1-R e^{i \lambda}\right|^{2}=\left|1-\rho e^{i \lambda}\right|^{2}-\frac{2 v}{n}\left(1-\rho^{2}\right)
$$

which determines the constants

$$
B=\frac{\rho}{R}
$$

and

$$
R=\frac{1+\rho^{2}-\frac{2 v}{n}\left(1-\rho^{2}\right) \pm i\left\{4 \rho^{2}-\left[1+\rho^{2}-\frac{2 v}{n}\left(1-\rho^{2}\right)\right]^{2}\right\}^{1 / 2}}{2 \rho}
$$

Introducing the angle $\theta$ by

$$
R=\cos \theta-i \sin \theta=e^{-i \theta}, \quad \cos \theta=\operatorname{Re} R=\frac{1+\rho^{2}-\frac{2 v}{n}\left(1-\rho^{2}\right)}{2 \rho},
$$

we get $\psi(z)=\log B$ so that

$$
\varphi_{\alpha}(z)=B^{-n / 2}=\left(\frac{R}{\rho}\right)^{n / 2}=\frac{e^{-i n \theta / 2}}{\rho^{n / 2}} .
$$


Inserting (24) into (22) we get

$$
g_{\alpha}(x)=-\frac{i}{2 \pi \rho^{m}} \oint e^{-\nabla x-i m \theta} d v,
$$

where we have supposed the sample size $n$ to be even, $n=2 m$. The above expression reduces to

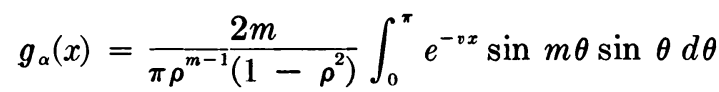

$$
=\frac{2 m e^{-\alpha x}}{\pi \rho^{m-1}\left(1-\rho^{2}\right)} \int_{0}^{\pi} e^{-\beta x \cos \theta} \sin m \theta \sin \theta d \theta
$$

with

$$
\left.\begin{array}{l}
\alpha=m \frac{1+\rho^{2}}{1-\rho^{2}} \\
\beta=-\frac{2 m \rho}{1-\rho^{2}}
\end{array}\right\} .
$$

Hence

$$
\begin{aligned}
g_{\alpha}(x) & =\frac{m e^{-\alpha x}}{\pi \rho^{m-1}\left(1-\rho^{2}\right)} \int_{0}^{\pi} e^{-\beta x \cos \theta}[\cos (m-1) \theta-\cos (m+1) \theta] d \theta \\
& =-\frac{m e^{-\alpha x} i^{m+1}}{\rho^{m-1}\left(1-\rho^{2}\right)}\left[J_{m-1}(i \beta x)+J_{m+1}(i \beta x)\right] \\
& =-\frac{m e^{-\alpha x} i^{m+1}}{\rho^{m-1}\left(1-\rho^{2}\right)}\left[i^{m-1} I_{m-1}(\beta x)+i^{m+1} I_{m+1}(\beta x)\right] \\
& =(-1)^{m} \frac{m e^{-\alpha x}}{\rho^{m-1}\left(1-\rho^{2}\right)}\left[I_{m+1}(\beta x)-I_{m-1}(\beta x)\right] \\
& =\frac{2 m^{2} e^{-\alpha x}(-1)^{m+1}}{\rho^{m-1}\left(1-\rho^{2}\right) \beta} \frac{1}{x} I_{m}(\beta x) \\
& =\frac{m e^{-\alpha x}(-1)^{m}}{x \rho^{m}} I_{m}(\beta x) .
\end{aligned}
$$

As a numerical test of the accuracy of this Toeplitz approximation we have plotted (26) and the corresponding frequency functions of the exact distributions for $\rho=.5$ and $\rho=.3$ in Figs. 5 and 6 . Because of the relatively small sample size used in the computations, the approximation (26) was thought to be adequate only for small values $\rho$ (wide spectral windows); it turned out, however, to be surprisingly close, as seen in the figures, so that its validity may well extend to somewhat narrower windows than expected.

4. Discussion of the approximations. We have examined the behavior of the eigenvalues of the matrix product $R W$ and compared it to that of $f\left(\lambda_{0}\right) W$. Because of the limited scope of this numerical study, our conclusions are necessarily of a tentative nature. It seems that the approximation is better the larger the sample size, the narrower the spectral window and the flatter the spectral density. Obviously, the spectral window has to be taken narrow enough not to smooth out too much of the large scale structure 
of the spectrum. If the time constant corresponding to this spectral window is only a fraction of $n$ we would expect this approximation to be satisfactory.

The Toeplitz approximation (26) seems to be valid if the time constant $\rho / 1-\rho$ is small compared to $n$.

Acknowledgment. We would like to thank Miss Cornelia M. Kalkman for her assistance with the numerical work.

\section{REFERENCES}

1. U. Grenander and M. Rosenblatt, Statistical analysis of stationary time series, John Wiley \& Sons, New York, 1957

2. U. Grenander and G. Szegö, Toeplitz forms and their applications, University of California Press, Berkeley and Los Angeles, 1958

3. U. Grenander, H. O. Pollak and D. Slepian, The distribution of quadratic forms in normal variates: A small sample theory with applications to spectral analysis, (to be published)

4. D. Slepian, Fluctuations of random noise power, The Bell System Tech. J. 37, 163-184 (Jan. 1958)

5. J. J. Kalker, IBM 650 programs for matrix computations based on Jacobi's method, Brown University Rept. DA-SC-78130/1, 1958 